\title{
NUDT15 genotyping in Caucasian patients can help to optimise thiopurine treatment in patients with inflammatory bowel disease
}

\author{
Marieke J. H. Coenen \\ Radboud University Medical Center, Radboud Institute for Health Sciences, Department of Human Genetics, Nijmegen, The Netherlands \\ Correspondence to: Marieke J. H. Coenen. Radboud University Medical Center, Radboud Institute for Health Sciences, Department of Human \\ Genetics, Internal mail 855, P.O. box 9101, 6500 HB Nijmegen, The Netherlands. Email: Marieke.Coenen@radboudumc.nl. \\ Provenance: This is an invited article commissioned by the Academic Editor Dr. Wei Liu, PhD. (Department of Gastroenterology of Yichang Central \\ People's Hospital, Institute of Digestive Disease, China Three Gorges University, Yichang, China). \\ Comment on: Walker GJ, Harrison JW, Heap GA, et al. Association of Genetic Variants in NUDT15 With Thiopurine-Induced Myelosuppression in \\ Patients With Inflammatory Bowel Disease. JAMA 2019;321:773-85.
}

Received: 17 October 2019; Accepted: 08 November 2019; Published: 12 December 2019.

doi: $10.21037 /$ tgh.2019.11.09

View this article at: http://dx.doi.org/10.21037/tgh.2019.11.09

Thiopurines [azathioprine (AZA) and 6-mercaptopurine (6$M P)]$ are frequently used for the treatment of inflammatory bowel disease (IBD). Unfortunately $~ 10-30 \%$ of the patients develop adverse drug reactions (1). Myelosuppression is one of the thiopurine related side-effects observed in $~ 5 \%$ of IBD patients from European descent (2-4). The course of thiopurine-induced myelosuppression is relatively mild but patients with this side-effect are more prone to serious infections. It is known that genetics can explain part of the thiopurine-induced myelosuppression.

Thiopurines need to undergo extensive metabolization to the active metabolite 6 -thioguanine nucleotides (6-TGN) (5). Already since 1980, it is known that genetic variants in the gene coding for thiopurine-Smethyltransferase (TPMT), one of the enzymes in the pathway results in an increased 6-TGN production (6). Indeed, subsequent studies showed that these genetic variants were linked to an increased risk of myelosuppression $(7,8)$. Interestingly, in the Asian population thiopurine induced myelosuppression is not explained by genetic variants in TPMT as these variants have a very low frequency in this population (3\% in Asians versus $10 \%$ in Caucasians) whereas the occurrence of thiopurine-induced myelosuppression is much higher in Asians (30-40\%) $(9,10)$. A few years ago a hypothesis free genome-wide association analysis (GWAS) was performed to identify genes linked to thiopurine-induced myelosuppression in Korean patients with IBD. The authors identified a statistically significant association between thiopurine-induced myelosuppression and a genetic variant in the nudix hydrolase 15 (NUDT15) gene (11). This variant results in an amino acid substitution at position 139 of the protein p.Arg139Cys, leading to an absence of NUDT15 enzyme activity (12). NUDT15 hydrolysis the cytotoxic thioguanine triphosphate to the less active thioguanine monophosphate. A decreased activity of NUDT15 results in more incorporation of thioguanine triphosphate into the DNA and consequently more damage related apoptosis and toxicity. Indeed, patients carrying the p.Arg139Cys variant in NUDT15 developed severe myelosuppression (12).

A second GWAS focussing on patients with acute lymphoblastic leukaemia (ALL) treated with thiopurines substantiated the link between NUDT15 and thiopurine induced myelosuppression (13). This US study once more confirmed that the genetic variant (risk allele) is present in a relatively high frequency in patients of East Asian descent. They observed lower allele frequencies of the NUDT15 p.Arg139Cys risk variant in Hispanics (3.9\%), very low frequencies in Europeans $(0.2 \%)$ and the variant was not observed in Africans. This suggests that thiopurine-induced myelosuppression related to genetic variants in the NUDT15 gene is mainly relevant for those populations with a high frequency of the risk allele. This population difference in frequency of the variant and thus the contribution of genes to the development of thiopurine-induced leukopenia was the starting point of the study by Walker et al.: Association of genetic variants in NUDT15 with thiopurine-induced myelosuppression in patients with IBD (14). The aim of 
the study was to investigate genetic variants contributing to thiopurine induced myelosuppression in patients from European descent.

Walker et al. used an original approach; investigation of common genetic variants (GWAS) was combined with more rare variants (exome-wide association analysis) (14). The authors first performed a GWAS including 311 patients with thiopurine-induced myelosuppression and 608 patients treated with thiopurines without myelosuppression. This analysis resulted in the identification of a genome wide significant signal in the TPMT gene. The top associated variant (rs11969064) was linked to an increased risk of myelosuppression $[\mathrm{OR}=2.3 ; 95 \%$ confidence interval (CI), 1.7-3.1]. These data confirm our knowledge that genetic variants in TPMT are linked to the development of thiopurine-induced myelosuppression $(6,15,16)$. As in previous papers this suggests that based on analysis of only common variants TPMT is the main predictor for thiopurine-induced myelosuppression in Caucasians. Rare variants were analysed, in 328 patients with thiopurineinduced myelosuppression and 608 patients treated with thiopurines without myelosuppression with next generation sequencing of all coding genes in the genome (whole exome sequencing) (14). A genetic association analysis using these data (exome-wide association analysis) also pointed to TPMT as predictor for thiopurine-induced myelosuppression. In addition, a statistically significant association was found for a genetic variant in NUDT15. The exome-wide association analysis was essential to pinpoint the contribution of NUDT15 to thiopurineinduced myelosuppression in a Caucasian population. From previous publications it was clear that genetic variants in the NUDT15 gene are rare in the Caucasian population. Therefore in order to find an association with these variants it was essential to sequence the complete NUDT15 gene as association with rare variants is difficult to detect using a genome-wide association approach as such variants are not well tagged on the arrays. Not only for this study but also for other studies focussing on multifactorial traits it can be of great value to investigate rare and common genetic variants combined to get a more complete picture of the genes contributing to the trait. Walker et al. investigated a Caucasian population and were able to identify rare genetic variants associated with thiopurine induced myelosuppression known to be associated with the trait in an Asian population (14). Applying similar approaches to other populations might make the genetic basis of thiopurine-induced myelosuppression even more complete.
But often such approaches are challenging as most (disease) cohorts collected focussed on specific populations (mainly Caucasians).

Interestingly, for both the TPMT and NUDT15 genes the authors found that the variants were overrepresented in persons with early myelosuppression ( $\leq 8$ weeks of thiopurine initiation) compared to persons that developed myelosuppression later after treatment initiation. Suggesting that other factors might play a role in late onset myelosuppression. The median time to onset of myelosuppression was 20 weeks [interquartile range (IQR) 7.6-48.3 weeks] for patients without a genetic variant in TPMT or NUDT15, patients with one genetic variant in TPMT have a lower time to onset but this was not significant [13.9 weeks (IQR 5.9-40.4 weeks)]. For patients with a variant in NUDT15 the earlier onset of myelosuppression was more pronounced [7.7 weeks (IQR 5.7-20.0 weeks), $\mathrm{P}=0.009]$. Patient homozygous for a genetic variant in TPMT had a lower time to myelosuppression compared to patients heterozygous for either TPMT or NUDT15 [6.1 weeks (range, 4.2-7.6 weeks)] whereas patients with a variant in both TPMT and NUDT15 developed a myelosuppression fastest [2.5 weeks (range, 1.5-4.1 weeks)]. Based on the thiopurine metabolism pathway this earlier onset of myelosuppression in patients with variants in both genes makes sense. TPMT and NUDT15 exert their effect at different places in the thiopurine metabolism pathway, based on their function it is expected that they have a synergistic effect (5). Genetic variants resulting in a decreased TPMT activity will increase the amount of medication that is converted to the active metabolite 6-TGN. NUDT15 is involved in the conversion of active metabolites to inactive metabolites and thereby preventing incorporation of the active metabolites in the DNA and RNA. A genetic variant resulting in a decreased function of the NUDT15 enzyme will thus result in higher levels of the active metabolites. Therefore it is expected that persons with a genetic variant in both genes (TPMT and NUDT15) will have faster accumulation of the active metabolite compared to persons with a genetic variant in only one of the genes. Suggesting that patients with variants in both genes should be treated with more caution.

The effect of the genetic variants in NUDT15 on thiopurine-induced myelosuppression is also reflected in the thiopurine dose that patients with a genetic variant can tolerate $(11,13)$. In patients with ALL it is shown that persons heterozygous for the variant could tolerate a lower thiopurine dose $[63 \%(n=31)]$ than patients without the variant $[83.5 \%(\mathrm{n}=624)]$. The tolerated dose was, as 
expected, even lower for patients homozygous for the variant $[8.3 \%(n=2)](13)$. These results are in line with a study investigating the effect of the genetic variant on the incorporation of thioguanine into the DNA (17). A significant positive correlation was observed between the DNA incorporated thioguanine in peripheral blood leucocytes with 6-MP dosage. In addition, 6-mercatopurine metabolism efficacy increased significantly with the number of NUDT15 defective alleles. In this same study, a mouse model was established by introduction of a single nucleotide insertion in Nudt15 that abolishes the translation of mouse NUDT15 protein. Mice homozygous for the genetic variant (Nudt15-/-) developed more toxicity and earlier death after exposure to 6-MP compared to wild-type mice (Nudt15+/+). Lowering 6-MP dose in Nudt15-/- mice significantly reduced the observed toxicity. In this mouse model leukaemia was induced, treatment with a low 6-MP dose showed that Nudt15-/- were completely leukaemia free for the duration of the experiment whereas this dose only had a minor effect on time to leukaemia onset in wildtype mice. Wild-type mice treated with the full 6-MP dose were also leukaemia free during the complete duration of the experiment. All these data is in line with the observation that patients with a genetic variant in NUDT15 develop thiopurine-induced myelotoxicity and suggest that patients with these genetic variants can be treated with a lower dose. Most studies have been performed in patients with leukaemia but it is not expected this will be different for patients with IBD.

Three commonly observed variants in NUDT15 p.Arg139Cys (rs116855232), p.Gly17_Val18del (rs746071566) and p.Val18Ile (rs186364861) were recently evaluated in a meta-analysis for their relation with thiopurine-induced myelosuppression in patients with IBD (18). The meta-analysis includes the results from the paper of Walker et al. for the first two variants evaluated (14). For p.Arg139Cys, the authors could include thirteen studies (1,136 variant carriers versus 4987 patients without the variant) and showed that persons with the variant had an statistically significant increased risk to develop thiopurine-induced myelosuppression (OR 6.9; 95\% CI, 5.2-9.1). Also for the other variants significant association were observed between the variants and thiopurine induced myelosuppression. For NUDT15 p.Gly17_Val18del an OR of 5.6 (95\% CI, 2.8-11.4) was observed (97 variant carriers and 906 patients without a variant in NUDT15) and for NUDT15 p.Val18Ile an OR of 5.6 (95\% CI, 2.8-11.4, 19 patients with the variant and 862 persons without the variant). Thus for these three variants the association with thiopurine-induced myelosuppression seems clear. However, more variants have been identified with a predicted deleterious effect on NUDT15 function. For instance, Walker et al. identified 7 of such variants and other studies in Caucasians have identified even more of such very rare variants $(14,19)$. As some variants occur in only 1 or a few patients it is difficult to assess their contribution to thiopurine-induced myelosuppression. Therefore in the paper of Walker they only focussed on variants that met genome-wide significance in their study or variants that were associated with thiopurine-induced myelosuppression in other studies (14). For the variants that were not investigated in detail it is also highly likely that they will have an effect NUDT15 protein function suggesting that patients carrying such variants will be at higher risk to develop thiopurine-induced myelosuppression. Another study, investigating 107 patients (including 68 patients with IBD) of European descent with very severe thiopurineinduced myelosuppression identified ten genetic variants in NUDT15 after sequencing the coding region of the gene (19). Three variants were also identified by Walker et al. (p.Gly17_ Val18del, p.Val18_Val19insGlyVal, p.Arg139Cys) (14). Five of the ten variants were considered (Based on the effect of the variant on NUDT15 protein function) to be relevant for thiopurine-induced myelosuppression. Indicating that by analysing more persons for genetic variation in NUDT15 even more rare genetic variants will be identified. This is not unique for NUDT15 e.g., for TPMT also rare variants have been identified in patients with thiopurine induced myelosuppression $(20,21)$.

Based on the study of Walker et al. and also previous literature it is clear that genetic variation in the NUDT15 gene also contributes to thiopurine-induced myelosuppression in patients from non-Asian descent $(14,19)$. In addition substantial evidence indicates that patients with a genetic variant in NUDT15 might be treated safer whit a decreased thiopurine dose (12). Recently the Clinical Pharmacogenetics Implementation Consortium (CPIC) and the Dutch Pharmacogenetics working group published guidelines regarding thiopurine dosing based on TPMT and NUDT15 pharmacogenetics $(22,23)$. Thus far 19 variants/variant combinations (haplotypes) have been reported in by the Pharmacogene Variation Consortium (PharmVar), but the effect of these on thiopurine-induced myelosuppression is not established firmly for all haplotypes (24). CPIC follows for NUDT15 genotype based dosing the recommendations for TPMT as the dose a patient with a genetic variant in NUDT15 can tolerate is highly similar to the dose patients with TPMT variants can tolerate. For 
patients with a genetic variant in one of the NUDT15 alleles it is recommended to start with a dose between $30-80 \%$ of normal whereas patients, with non-malignant conditions (like IBD), homozygous for a genetic variant in NUDT15 are advised to start with an alternative treatment (22). The guidelines also indicate to even further reduce the thiopurine dose when patients carry a genetic variant in both TPMT and NUDT15. However, the evidence for these recommendations is only limited as only a few patients carry a genetic variant in both genes have been studied. In the paper of Walker et al. it is recommended to incorporate pharmacogenetic testing for NUDT15 in the clinical setting for patients of European ancestry. Their study showed that the number needed to genotype to avoid one case of thiopurine-induced myelosuppression is 95 , which is similar to the number needed to genotype for TPMT. The authors recommend to sequence the gene including the p.Gly17_Val18del. From this statement it is not completely clear whether they suggest to analyse the complete gene or the relevant variants included in their subsequent analysis (p.Gly17_Val18del, p.Gly17_ Val18dup and p.Arg139Cys). Sequencing a complete gene for diagnostics is relatively costly and will also lead to the identification of genetic variants with an unknown effect, which will make it difficult to determine an appropriate dose for such patients. Thus analysing variants with a known effect for which dosing guidelines are available might be a better choice for translation of the results to the clinical setting. Of course, there is a risk that a variant will be missed but even though the thiopurine dose is adjusted based on genotype patients still need to be monitored closely for the development of thiopurine-induced myelosuppression as the two major genes involved in this process do not explain all cases of this side-effect.

Walker et al. confirmed that genetic variants in NUDT15 also contributes to thiopurine-induced myelosuppression in Caucasians (14). Their data also indicate that TPMT and NUDT15 are the genes contributing most to thiopurineinduced myelosuppression. However, other genes contributing to thiopurine-induced might be identified when analysing larger patient groups.

\section{Acknowledgments}

None.

\section{Footnote}

Conflicts of Interest: The author has no conflicts of interest to declare.

Ethical Statement: The author is accountable for all aspects of the work in ensuring that questions related to the accuracy or integrity of any part of the work are appropriately investigated and resolved.

\section{References}

1. Chaparro M, Ordas I, Cabre E, et al. Safety of thiopurine therapy in inflammatory bowel disease: long-term follow-up study of 3931 patients. Inflamm Bowel Dis 2013;19:1404-10.

2. Present DH, Meltzer SJ, Krumholz MP, et al. 6-Mercaptopurine in the management of inflammatory bowel disease: short- and long-term toxicity. Ann Intern Med 1989;111:641-9.

3. Connell WR, Kamm MA, Ritchie JK, et al. Bone marrow toxicity caused by azathioprine in inflammatory bowel disease: 27 years of experience. Gut 1993;34:1081-5.

4. Fraser AG, Orchard TR, Jewell DP. The efficacy of azathioprine for the treatment of inflammatory bowel disease: a 30 year review. Gut 2002;50:485-9.

5. Zaza G, Cheok M, Krynetskaia N, et al. Thiopurine pathway. Pharmacogenet Genomics 2010;20:573-4.

6. Weinshilboum RM, Sladek SL. Mercaptopurine pharmacogenetics: monogenic inheritance of erythrocyte thiopurine methyltransferase activity. Am J Hum Genet 1980;32:651-62.

7. Coenen MJ, de Jong DJ, van Marrewijk CJ, et al. Identification of Patients With Variants in TPMT and Dose Reduction Reduces Hematologic Events During Thiopurine Treatment of Inflammatory Bowel Disease. Gastroenterology 2015;149:907-17.e7.

8. Evans WE, Horner M, Chu YQ, et al. Altered mercaptopurine metabolism, toxic effects, and dosage requirement in a thiopurine methyltransferase-deficient child with acute lymphocytic leukemia. J Pediatr 1991;119:985-9.

9. Kim JH, Cheon JH, Hong SS, et al. Influences of thiopurine methyltransferase genotype and activity on thiopurine-induced leukopenia in Korean patients with inflammatory bowel disease: a retrospective cohort study. J Clin Gastroenterol 2010;44:e242-8.

10. Takatsu N, Matsui T, Murakami Y, et al. Adverse reactions to azathioprine cannot be predicted by thiopurine S-methyltransferase genotype in Japanese patients with inflammatory bowel disease. J Gastroenterol Hepatol 
2009;24:1258-64.

11. Yang SK, Hong M, Baek J, et al. A common missense variant in NUDT15 confers susceptibility to thiopurineinduced leukopenia. Nat Genet 2014;46:1017-20.

12. Moriyama T, Nishii R, Perez-Andreu V, et al. NUDT15 polymorphisms alter thiopurine metabolism and hematopoietic toxicity. Nat Genet 2016;48:367-73.

13. Yang JJ, Landier W, Yang $W$, et al. Inherited NUDT15 variant is a genetic determinant of mercaptopurine intolerance in children with acute lymphoblastic leukemia. J Clin Oncol 2015;33:1235-42.

14. Walker GJ, Harrison JW, Heap GA, et al. Association of Genetic Variants in NUDT15 With Thiopurine-Induced Myelosuppression in Patients With Inflammatory Bowel Disease. JAMA 2019;321:773-85.

15. Liu C, Yang W, Pei D, et al. Genomewide Approach Validates Thiopurine Methyltransferase Activity Is a Monogenic Pharmacogenomic Trait. Clin Pharmacol Ther 2017;101:373-81.

16. Tamm R, Magi R, Tremmel R, et al. Polymorphic variation in TPMT is the principal determinant of TPMT phenotype: A meta-analysis of three genomewide association studies. Clin Pharmacol Ther 2017;101:684-95.

17. Nishii R, Moriyama T, Janke LJ, et al. Preclinical evaluation of NUDT15-guided thiopurine therapy and its effects on toxicity and antileukemic efficacy. Blood

doi: 10.21037/tgh.2019.11.09

Cite this article as: Coenen MJ. NUDT15 genotyping in Caucasian patients can help to optimise thiopurine treatment in patients with inflammatory bowel disease. Transl Gastroenterol Hepatol 2019;4:81.
2018;131:2466-74.

18. van Gennep S, Konte K, Meijer B, et al. Systematic review with meta-analysis: risk factors for thiopurineinduced leukopenia in IBD. Aliment Pharmacol Ther 2019;50:484-506.

19. Schaeffeler E, Jaeger SU, Klumpp V, et al. Impact of NUDT15 genetics on severe thiopurine-related hematotoxicity in patients with European ancestry. Genet Med 2019;21:2145-50.

20. Ujiie S, Sasaki T, Mizugaki M, et al. Functional characterization of 23 allelic variants of thiopurine S-methyltransferase gene (TPMT*2 - *24). Pharmacogenet Genomics 2008;18:887-93.

21. Schaeffeler E, Eichelbaum M, Reinisch W, et al. Three novel thiopurine $\mathrm{S}$-methyltransferase allelic variants $\left(\mathrm{TPMT}^{*} 20,{ }^{*} 21,{ }^{*} 22\right)$ - association with decreased enzyme function. Hum Mutat 2006;27:976.

22. Relling MV, Schwab M, Whirl-Carrillo M, et al. Clinical Pharmacogenetics Implementation Consortium Guideline for Thiopurine Dosing Based on TPMT and NUDT15 Genotypes: 2018 Update. Clin Pharmacol Ther 2019;105:1095-105.

23. Available online: https://www.knmp.nl/downloads/ pharmacogenetic-recommendations-november-2019.pdf

24. Yang JJ, Whirl-Carrillo M, Scott SA, et al. Pharmacogene Variation Consortium Gene Introduction: NUDT15. Clin Pharmacol Ther 2019;105:1091-4. 